Héctor Ferreiro, Buenos Aires

\title{
Der praktische Geist ist der wirkliche Geist
}

\author{
Zu Hegels Antirepräsentationalismus
}

Hegel weist das Weltbild zurück, nach dem das erkennende Subjekt ein Ding unter den Dingen ist, das alle Dinge - dabei also auch sich selbst - kausal widerspiegelt, und schlägt stattdessen ein neues Paradigma vor, in dem das erkennende Subjekt als die Einheit der Erkenntnistätigkeit (des „Subjekts“ vom alten Paradigma) und des durch sie Erkannten (des „Objekts“) aufgefasst wird. Für diese neue Auffassung gehört das Objekt nicht zum Anderen der erkennenden Subjektivität, die es daher als ein Fremdes widerspiegelt; Objekt und Subjekt sind vielmehr strukturelle und strukturierende Momente des Erkennens bzw. des Denkens selbst, welches nun als der „Raum“ verstanden wird, in dem Objekt und Subjekt, Welt und Geist vorkommen. Das Ziel des vorliegenden Beitrags besteht darin, Hegels Ansatz gegen die repräsentationalistische Auffassung der Erkenntnis zu analysieren und in diesem Kontext den Sinn hervorzuheben, den seine These über die praktischen Tätigkeit als Wesen und Wahrheit der kognitiven Tätigkeit hat.

\section{Vom vorstellenden Einzelsubjekt zur intersubjektiven Tätigkeit des Begreifens}

Das Subjekt entwickelt für Hegel die Fähigkeit zur Sprache, wenn es die Bestimmungen der Außenwelt auf seine eigenen Bestimmungen reduziert, $d$. h. wenn es diesen Bestimmungen ihren sinnlichen Charakter abstreift, während es ihren Inhalt bzw. ihre Bestimmtheit behält. Das Subjekt, das diese Phase erreicht hat, stellt sich die wirkliche Welt nicht mehr in seinem „Inneren“ vor, sondern es bewegt sich in dem Raum der Vernünftigkeit, der weder lediglich objektiv noch lediglich subjektiv ist. In der der Subjektivität charakteristischen Idealität, welche trotzdem anhand der Sprache prinzipiell mit der konkreten Wirklichkeit identisch bzw. identifizierbar ist, hebt der menschliche Geist seine eigene, einseitige Subjektivität auf und wird zur einfachen Einheit des Objekts und seiner selbst als Subjekt. ${ }^{1}$ Die kognitive Tätigkeit des Geistes, der sich zu dieser Einheit erhoben hat und sich nunmehr in ihr bewegt, ist nicht mehr ein bloßes Sich-Vor-Stellen dessen, was sich aus sich selbst darstellt: Indem die Sprache den Subjekt-Objekt-Dualismus überwindet, ist sie die Möglichkeitsbedingung der dem Subjekt vollkommen immanenten und trotzdem vollkommen objektiven Tätigkeit des Begreifens. ${ }^{2}$ Das Begreifen besteht genau genommen darin, die Bestimmtheit jedes Objekts zu analysieren und ihre verschiedenen, konstitutiven Bestimmungen in ihrer Notwendigkeit aufeinander zu beziehen. Dadurch überwindet das Begreifen die Unmittelbarkeit und Undurchsichtigkeit der Inhalte, die in der Sprache zunächst sozusagen bloß „da sind“, d. h. es überwindet die schon idealisierte, aber immer noch in sich selbst äußerliche Bestimmtheit des durch die Sprache formulierten Objekts. In der Phase der Vorstellung überwindet der Erkenntnisprozeß die Äußerlichkeit der Bestimmungen im Verhältnis zum Geist als reinem Subjekt bzw. als Ich, während er in der Phase des Denkens die Äußerlichkeit der Bestimmungen zueinander

1 Vgl. G. W. F. Hegel, Enzyklopädie der philosophischen Wissenschaften im Grundrisse (1830), Hamburg 1992, §§ 465466.

2 Ebd., § 467. 
aufhebt. In der letzten Form des Denkens, d. h. im eigentlichen Begreifen erkennt der menschliche Geist die Wirklichkeit in ihrer innerlichen Struktur und enthüllt damit, dass jede Bestimmung der sogenannten Außenwelt in Wahrheit eine Selbstdifferenzierung der konstitutiven Prinzipien der Vernünftigkeit ist, die den Geist definieren: Der Geist ist nämlich für Hegel weder ein Ding unter anderen Dingen noch ein Vermögen eines Dings, sondern der Prozess der Selbstunterscheidung und Selbstvereinzelung des Systems des jeweiligen Theoretisierens über die Wirklichkeit bzw. des „Begriffs“.

Eben deshalb, weil der menschliche Geist sich durch das Begreifen als identisch mit den konstitutiven Prinzipien der Vernünftigkeit manifestiert, ist das, was er jeweils für die wirkliche Welt hält, eine Selbstdifferenzierung des Geistes selbst. Das Begreifen impliziert für Hegel insofern nicht nur die Selbstvereinzelung der allgemeinen Kategorien der Vernünftigkeit in der einzelnen Bestimmtheit des begriffenen Objekts, sondern zugleich die Selbstvereinzelung der subjektiven Vernunft, die das Begreifen vollbringt. Indem das Subjekt das Objekt begreift und alle Ebenen der Bestimmtheit desselben als eigene Bestimmungen setzt, offenbart es sich als die Einheit seiner selbst und des Objekts; damit erscheint das erkennende Subjekt nicht mehr als eine gegenüber der Welt leere Form, sondern es wird selbst zur wirklichen Welt.

Ist die Welt aber nicht zwangsläufig das Andere des Ichs, das sie begreift? Inwiefern kann also das Begreifen implizieren, dass die Welt, die begriffen wird, dadurch zu einer Selbstbestimmung der subjektiven Tätigkeit ihres Begreifens wird? Hegels Antwort auf diese Frage beruht auf der These, dass bereits die Inhalte, die sich das Subjekt in seinem Inneren als verschieden von den Objekten der Außenwelt vorstellt, nicht in ihm vor ihm stehen, sondern strenggenommen eigene Bestimmungen des Subjekts sind. Die vom Subjekt innerlich vorgestellte Bestimmtheit des Objekts ist für Hegel nämlich die Qualität, mit der das Subjekt, welches sich anfangs gegenüber dem bestimmten Objekt als in sich rein formell und leer auffasst, nunmehr explizit sich in sich selbst bestimmt. Deshalb hält Hegel die abstrakt allgemeine Vorstellung, in welcher der konkrete, sinnliche Charakter der wirklichen Welt (teilweise) verschwindet, für eine erste Manifestation der konstitutiven Idealität der Welt selbst bzw. für ein Anzeichen, dass die Wirklichkeit kein Bereich ist, in dem vom Subjekt völlig unabhängige Dinge äußerlich existieren. Das Bewusstwerden darüber, dass das Wirkliche auch nicht nur eine „innere“ Welt des Subjekts ist, hebt endgültig den einseitigen Standpunkt des Vorstellens auf und überwindet damit das allgemeine Paradigma der Subjektivität als verschieden von der objektiven Welt: Das Begreifen ist nämlich eine sowohl subjektive als auch objektive Tätigkeit.

Das Vorstellen überhaupt gilt in Hegels Erkenntnistheorie als die anfängliche Manifestation der Idealität der Bestimmtheiten, die das Subjekt zunächst als Dinge einer Außenwelt erkennt. Aber sogar in den abstrakten Vorstellungen unterscheiden sich immer noch deren einseitig subjektiver Inhalt und der einseitig objektive Inhalt der sinnlichen Anschauung voneinander; dabei setzt sich das Erkennen als subjektive Tätigkeit den nicht-subjektiven Inhalten als Dingen einer dem erkennenden Subjekt äußeren Welt entgegen. Im Unterschied zur bloß abstrakten Vorstellung enthält die Vorstellung der Bedeutung der Sprachzeichen in ihrer nicht-sinnlichen Allgemeinheit die bestimmte Einzelheit des konkreten, sinnlichen Objekts ohne seine sinnliche Konkretion. In dieser Hinsicht sagt Hegel: „Caesar ist längst vorbei, als Name hat er sich erhalten.“3 Und weiter: „Die Namen sind keine Bilder, und doch haben wir den ganzen Inhalt, indem wir den Namen vor uns haben. ${ }^{* 4}$ Indem die Vorstellung, die als die Bedeutung eines Namens fungiert, das bis dahin ausgeschlossene, sinnliche Objekt ohne seine ursprüngliche Sinnlichkeit in sich einschließt, bringt sie eine tiefere Idealisierung zustande, als die, die die bloß abstrakte Vorstellung zustande bringt; sie tut dies, indem die Bestimmungen des vorher nur abstrakt vorgestellten Inhalts durch die Sprache homogener mit der sie erkennenden Subjektivität werden; eben

3 G. W. F. Hegel, Vorlesungen über die Philosophie des Geistes. Berlin 1827/1828. Nachgeschrieben von J E. Erdmann und F. Walter, Hamburg 1994, 215.

4 Ebd., 218. 
deshalb kann das sprachfähige Subjekt über diese Bestimmungen verfügen und sie frei aufeinander beziehen. Anhand der Sprache gestaltet der Geist die Kategorien, durch die er die wirklichen Objekte begreifen kann. Diese Kategorien sind keine Vorstellungen mehr, sondern vielmehr - in Hegels Terminologie - „Gedanken“, d. h. immanente Veränderungen des Erkennens bzw. des Denkens selbst, weil sie die Aufhebung des Gegensatzes von erkanntem, äußerem Objekt und erkennendem, innerem Subjekt voraussetzen, der das Vorstellen als solches definiert. Die Kategorien und Gesetze - egal welche sie jeweils sind -, anhand derer der menschliche Geist die Welt begreift, sind sozusagen das innere Leben der Vernünftigkeit, die sich in ihrer völlig nicht-sinnlichen Idealität trotzdem als die Welt bestimmt bzw. die Welt ist. Indem das Begreifen jeden Inhalt aus den höchst ideellen Bestimmtheiten der allgemeinen Kategorien und Denkgesetze in sich vermittelt, überwindet es in Hegels Augen alle Ebenen der Andersheit des Inhalts, da die Kategorien und Denkgesetze keine Bestimmungen des menschlichen Geistes als dieses oder jenes einzelne Subjekt sind, sondern die logisch-ontologischen Grundprinzipien dessen, was der Geist jeweils für die Wirklichkeit hält. Das Begreifen ist für Hegel insofern das Sich-Urteilen und Sich-Zusammenschließen der Subjektivität - oder was dasselbe ist, die Vernünftigkeit selbst als tätiges und lebendiges Subjekt.

Der Geist, der die Wirklichkeit begreift, bestimmt sich für Hegel, wie vorausgeschickt wurde, in sich selbst mit den Bestimmungen des Wirklichen. Im Begreifen als Vollendung der Erkenntnistätigkeit sind die wirklichen Objekte Selbstbestimmungen des menschlichen Geistes. Dies bedeutet aber nicht, dass für Hegel das Objekt etwas Subjektives ist in dem Sinne, dass es keine wirkliche Welt gibt. Begreifen ist zwar eine subjektive, aber objektive Tätigkeit des einzelnen Subjekts. Fast vollständig gelähmt sprach der Physiker Stephen Hawking über Galaxien, die Millionen Lichtjahre von der Erde entfernt sind, und über Ereignisse, die vor Tausenden von Millionen Jahren geschehen sind; für die physikalische Theorie von Hawking waren wiederum die Wechselfälle seines Lebens völlig irrelevant: Wenn die subjektive Tätigkeit des Begreifens die Ebene der konstitutiven Bestimmungen der Wirklichkeit erreicht, hebt sie auch ihre eigene besondere Subjektivität auf. Was im Begreifen sich in sich selbst bestimmt, ist also nicht das einzelne Subjekt, das sowohl die Objekte als auch die anderen Subjekte aus sich ausschließt, sondern die Subjektivität, die sich als identisch mit der Wirklichkeit offenbart. Hegels These will darauf hinaus, dass das Erkennen die Substanz ist bzw. dass die Substanz Subjekt ist. ${ }^{5}$

Das Erkennen versteht sich zunächst als eine völlig formelle Tätigkeit und demnach als ein rein „ideelles“ Phänomen gegenüber dem Objekt, das seinerseits eben als das Andere des ideellen Erkennens für ein Ding der „wirklichen“ Welt gehalten wird. Wenn aber das Erkennen bzw. das Denken begreift, dass sein Inhalt eigentlich das Denken selbst ist, verliert es seinen formellen, ideellen Charakter und wird qua erkennende Tätigkeit wirklich. ${ }^{6}$ Die unmittelbare Schlußfolgerung dieser Bewusstwerdung ist, dass Erkennen in Wahrheit praktische Tätigkeit ist bzw., dass die Intelligenz in Wahrheit Wille ist - oder, aus einer anderen Perspektive, dass die Struktur des Objekts die eigene Struktur der Subjektivität ist. ${ }^{7}$

\section{Erkennen als praktisches Tun}

Das Erkennen, das seine Tätigkeit als reine Theorie und dementsprechend sich selber als eine in sich leere, abstrakt ideelle Form versteht, die ihre Inhalte von einer „Außen“-Welt erhält, offenbart am Ende seines Prozesses, dass das Erkennen in Wahrheit ein Kontinuum ist, innerhalb dessen

5 Vgl. Hegel, Enzyklopädie der philosophischen Wissenschaften, a. a. O. (Anm. 1), § 213, § 215 Anm., § 573 Anm. 6 Vgl. Hegel, Vorlesungen über die Philosophie des Geistes, a. a. O. (Anm. 3), 241. Siehe auch Hegel, Enzyklopädie, a. a. O. (Anm. 1), § 482.

7 Vgl. Hegel, Grundlinien der Philosophie des Rechts, Frankfurt am Main 1970, Bd. 7, § 4 Zusatz. 
das erkennende Subjekt und das erkannte Objekt, der Geist und die Welt, nur Momente sind. Eben deshalb charakterisiert Hegel den praktischen Geist bzw. den Willen als den „wirklichen Geist“, d. h. als die Gestalt des menschlichen Geistes, in der die Realität des Erkennens, das den Geist als solches definiert, seinem Begriff entspricht. ${ }^{8}$ Obwohl die These, dass das Praktische die Wahrheit des Theoretischen ist, innerhalb von Hegels Philosophie eine entscheidende Rolle spielt, hat man ihr selten Aufmerksamkeit beigemessen. Hegel selbst hat womöglich dazu beigetragen, da er seine Philosophie des (subjektiven) Willens nicht in großem Detail ausgeführt hat. ${ }^{9}$

Der spezifische Prozess des Willens besteht für Hegel darin, die Gegebenheit und Unmittelbarkeit der eigenen Selbstbestimmungen des menschlichen Geistes aufzuheben. Die erste Phase dieser Aufhebung ist das Sich-Teilen des Selbstbewusstseins als Tätigkeit, d. h. seine reelle Ur-Teilung. ${ }^{10}$ Das „objektive“ Extrem dieses Sichteilens der unmmittelbaren Tätigkeit des Selbstbewusstseins ist ihre Verallgemeinerung zu einem (teilweise) allgemeinen Inhalt, nämlich zu den Trieben und Neigungen des Geistes; das „subjektive“ Extrem ist die rein abstrakte Reflexion-in-sich derselben Tätigkeit des Selbstbewusstseins nun als Form in Bezug auf sich selbst als (teilweise verallgemeinerten) Inhalt: Dieses subjektive, formelle Extrem des Sichteilens des wirklichen Geistes ist nichts anderes als seine Fähigkeit bzw. seine Macht zum Wählen seiner eigenen Triebe und Neigungen, d. h. die Willkür. ${ }^{11}$ Indem der wirkliche Geist sich in sich teilt, reproduziert er gewissermaßen die theoretische Trennung zwischen Objekt und Subjekt; aber dadurch, dass die Objekt-Subjekt-Dualität aufgehoben ist und somit das „Objekt“ bzw. der Inhalt nun ausdrücklich selbst Subjektivität ist - die Triebe und Neigungen sind nämlich Selbstbestimmungen des Geistes -, ist die Beziehung zwischen beiden Extremen des Sichteilens des Geistes auf dieser Ebene keine theoretische, sondern eine praktische Beziehung: Diese Beziehung ist das tätige Wählen selbst, d. h. der Entschluß, durch welchen der Geist nach seiner Selbstteilung sich mit sich wieder zusammenschließt. ${ }^{12}$

Die nicht mehr abstrakt ideelle, sondern als solche wirkliche Tätigkeit des Wissens - d. h. der Wille - wird sich im subjektiven Extrem ihres Sich-Teilens ihrer eigenen Formalität bewusst (Willkür), und zwar diesmal ihrer Formalität hinsichtlich ihrer selbst qua Inhalt, d. h. hinsichtlich ihrer eigenen Selbstbestimmungen (Triebe und Neigungen). Sowohl die Triebe und Neigungen als auch die Willkür sind aber besondere Momente des wirklichen Geistes, weil sie als solche voneinander verschieden sind und ihrem Begriff nach verschieden bleiben; ihre Allgemeinheit ist deshalb immer nur abstrakt: Sie schließt das Moment der Besonderheit nicht in sich ein, weshalb das jeweils andere Extrem trotz aller möglichen, gegenseitigen Vermittlung (die Vermittlung beider Extreme des wirklichen Geistes ist der jeweilige Entschluß) in seiner eigenen Besonderheit verharrt.

Die Besonderheit der zwei entgegengesetzten Momente des Willens wird für Hegel aufgrund der theoretischen Form aufgehoben, durch die der wirkliche Geist sich erkennt. Im eigentlichen theoretischen Prozess lassen sich Objekt und Subjekt nicht als solche aufeinander reduzieren; ihre Dualität kann nämlich als solche nicht aufgelöst werden: Es gibt immer ein Objekt, das erkannt

8 Vgl. Hegel, Vorlesungen über die Philosophie des Geistes, a. a. O. (Anm. 3), 241.

$9 \mathrm{Zu}$ einer detaillierten Analyse von Hegels Theorie des subjektiven Willens siehe u. a. Adriaan Peperzak, Modern Freedom, Dordrecht/Boston/London 2001, 174-211; Héctor Ferreiro, „Reconstrucción del sistema de la voluntad en la filosofía de Hegel“, in: Revista Latinoamericana de Filosofía 35, 2 (2009), 55-86.

10 Vgl. Hegel, Enzyklopädie der philosophischen Wissenschaften, a. a. O. (Anm. 1) § 473; Hegel, Vorlesungen über die Philosophie des subjektiven Geistes. Nachschriften zu den Kollegien der Jahre 1822 und 1825, Hamburg 2008, 537; Hegel, Vorlesungen über die Philosophie des Geistes, a. a. O. (Anm. 3), 251.

11 Vgl. Hegel, Enzyklopädie der philosophischen Wissenschaften, a. a. O. (Anm. 1) § 477; Hegel, Vorlesungen über die Philosophie des subjektiven Geistes, a. a. O. (Anm. 10), 540; Hegel, Vorlesungen über die Philosophie des Geistes, a. a. O. (Anm. 3), 256-258. Siehe auch Hegel, Grundlinien der Philosophie des Rechts, Hamburg 2009, § 14 Anm., § 15.

12 Vgl. Hegel, Enzyklopädie der philosophischen Wissenschaften, a. a. O. (Anm. 1) § 478; Hegel, Vorlesungen über die Philosophie des Geistes, a. a. O. (Anm. 3), 256; Hegel, Grundlinien der Philosophie des Rechts, Hamburg 2009, §13, §13 Anm., $§ 16$. 
wird, und ein Subjekt, das es erkennt. Das Erkennen als bloßes Sich-aufeinander-Beziehen von Objekt und Subjekt ist in sich rein formell; die Aufhebung der Besonderheit beider Extreme findet insofern auf der Ebene ihrer Bestimmtheit statt; wenn die Bestimmtheit auf beiden Seiten vollkommen identisch ist, sind Objekt und Subjekt trotz ihres unüberwindbaren Unterschieds keine besonderen Momente mehr, sondern sie bilden eine in sich differenzierte Identität. Auf eine ähnliche Weise ist der Entschluß als bloßes Sich-aufeinander-Beziehen der beiden Extreme des Willens - d. h. der Willkür und der Triebe und Neigungen - nicht imstande, die Besonderheit dieser Extreme aufzuheben. Es ist der Entschluß aufgrund der Erkenntnis dessen, was der wirkliche Geist in Wahrheit ist, der die Besonderheit der Triebe und der Willkür aufhebt. Wenn der wirkliche Geist begreift, dass seine wahre Natur weder eine Summe von Trieben und Neigungen noch die reine Macht zum Wählen, sondern die konkrete Einheit von beiden ist, kann er diese Einheit als die wahre Bestimmtheit bzw. als das Endziel seiner Entschlüsse setzen. Der Geist, der sich als eine in sich differenzierte Einheit von Trieben und Wahlfähigkeit erkennt und sich zu dieser Einheit als Endziel entschließt, ist als nunmehr konkret allgemeiner, wirklicher Geist sozusagen zweimal wirklicher Geist, wirklich freier Geist. ${ }^{13}$

Was bedeuten aber diese auf den ersten Blick undurchschaubaren Thesen von Hegel über den menschlichen Willen? Und vor allem: Inwiefern betreffen sie die Erkenntnistheorie? Wie oben ausgeführt, ist die praktische Tätigkeit für Hegel das eigentliche Wesen der theoretischen Tätigkeit. Diese These bedeutet genauer, dass die Erkenntnistätigkeit, durch die die Bestimmtheit, die zunächst als ein unmittelbares Objekt erscheint, auf ein Moment der allgemeinen Welttheorie des Subjekts reduziert wird, von einem formellen Standpunkt aus dieselbe Tätigkeit ist, durch welche der Geist seine zunächst unmittelbaren Selbstbestimmungen auf sich als nun in Bezug auf diese Selbstbestimmungen gesetzte Form - d. h. als fähig, sie zu wählen - reduziert. Noch deutlicher ausgedrückt: Für Hegel kann das Subjekt im Erkenntnisprozess alle Bestimmungen, die es erkennt, miteinander vermitteln und dadurch die Inhalte begreifen, die es zunächst als äußere Dinge anschaut und sich dann als bloß innere Inhalte vorstellt, eben weil das Subjekt seine als jene Inhalte bestimmten Anschauungs- und Vorstellungsakte auf Momente seiner selbst als wirklich freies Subjekt reduziert. Die Allgemeinheit ist die Eigenschaft eines Inhalts, mit anderen Inhalten vermittelt zu werden; in Wahrheit ist diese Eigenschaft und die gegenseitige Vermittlung der Inhalte für Hegel aber jeweils eine Fähigkeit und eine Tätigkeit des menschlichen Geistes, der diese Inhalte erkennt und eben deshalb dann begreifen kann, weil er mit ihrer Qualität in sich bestimmt ist, dabei aber auch selber wirklich frei ist. In diesem Zusammenhang kann Hegel daher behaupten: „Der praktische Geist hat nicht nur Ideen, sondern ist die lebendige Idee selbst.“14

\section{Schlußbemerkungen}

Die Sprachzeichen sind Inhalte, die der Geist in der Idealität seiner Denktätigkeit erkennt, ohne sie zu Außendingen zu machen. Die Sprache ist insofern das spezifische Element der Vernünftigkeit, in der das Objekt sich nicht einem Subjekt darstellt, das sich wiederum dieses Objekt vorstellt. Was der Mensch sprachlich formuliert, gehört ihm nicht als diesem besonderen Subjekt, sondern es gehört dem Menschenwesen ohne weiteres; das vom Einzelsubjekt sprachlich Formulierte ist seinerseits kein Ding einer dem Subjekt äußeren Welt, sondern als solches ein Inhalt, der in den logischen Raum der Gründe anderer sprachfähiger Subjekte integriert ist. Weder das Subjekt noch das Objekt sind für Hegel Dinge unter Dingen. Als die konkrete Einheit von beiden ist das begreifende Erkennen der eigentliche Raum, in dem alles Wirkliche als Moment des Erkennens erscheint. Hegel fasst das Erkennen aber nicht als eine rein ideelle Tätigkeit, sondern als

13 Vgl. Hegel, Enzyklopädie, a. a. O. (Anm. 1), §§ 480-481.

14 G. W. F. Hegel, Nürnberger und Heidelberger Schriften (1808-1817), Frankurt am Main 1970, Bd. 4, 57. 
wirklichen Geist, d. h. als praktische Tätigkeit auf. Die These, dass die theoretische Tätigkeit in Wahrheit praktische Tätigkeit bzw., dass die Intelligenz Wille ist, krönt Hegels philosophische Anthropologie bzw. seine Philosophie des subjektiven Geistes; trotzdem ist sie in der Regel sogar von denjenigen übersehen worden, die versuchen, Hegels Erkenntnistheorie unter besonderer Berücksichtigung der Rolle zu rekonstruieren, die in dieser Erkenntnistheorie die praktische Dimension des Menschenlebens für die Konstitution der Vernünftigkeit spielen soll. ${ }^{15}$ Mit dem vorliegenden Beitrag wollte ich in erster Linie auf diesen Mangel in der Hegel-Forschung aufmerksam machen und dabei einen Beitrag dazu leisten, ihn zu beheben.

Prof. Dr. Héctor Ferreiro

Consejo Nacional de Investigaciones Científicas y Técnicas (CONICET)

Pontificia Universidad Católica Argentina

Alicia Moreau de Justo 1500 (C1107AFD)

Buenos Aires, Argentina

hferreiro@conicet.gov.ar

15 Vgl. in diesem Sinne z. B. Thomas Auinger, „Praxis und Objektivität: Anmerkungen zu Robert Brandoms postanalytischer Hegel-Interpretation“, in: Internationales Jahrbuch des Deutschen Idealismus, Bd. 3, Deutscher Idealismus und die analytische Philosophie der Gegenwart, hg. v. K. Ameriks und J. Stolzenberg, Berlin/New York 2005, 162-178; Terry Pinkard, „Was Pragmatism the Sucessor to Idealism?““, in: New Pragmatists, hg. v. C. Misak, New York 2007, 142-168; Michael Barber, The Intentional Spectrum and Intersubjectivity: Phenomenology and the Pittsburgh Neo-Hegelians, Athens 2011, 87-127. 